\title{
Absorbing and shattered fragmentation transitions in multilayer coevolution
}

\author{
Marina Diakonova, Maxi San Miguel, and Víctor M. Eguíluz \\ Instituto de Física Interdisciplinar y Sistemas Complejos (CSIC-UIB), E-07122 Palma de Mallorca, Spain
}

(Received 14 March 2014; published 30 June 2014)

\begin{abstract}
We introduce a coevolution voter model in a multilayer by coupling a fraction of nodes across two network layers (the degree of multiplexing) and allowing each layer to evolve according to its own topological temporal scale. When these time scales are the same, the time evolution equations can be mapped to a coevolution voter model in a single layer with an effective average degree. Thus the dynamics preserve the absorbing-fragmentation transition at a critical value that increases with the degree of multiplexing. When the two layers have different topological time scales, we find an anomalous transition, named shattered fragmentation, in which the network in one layer splits into two large components in opposite states and a multiplicity of isolated nodes. We identify the growth of the number of components as a signature of this anomalous transition. We also find the critical level of interlayer coupling needed to prevent the fragmentation in a layer connected to a layer that does not fragment.
\end{abstract}

DOI: 10.1103/PhysRevE.89.062818

PACS number(s): 64.60.aq, 89.75.Hc, 89.75.Fb

\section{INTRODUCTION}

The general framework linking together networks that represent different processes is that of a multilayer system [1,2]. Its significance has recently been highlighted in situations ranging from infrastructure [3], information transmission, and epidemic spreading $[4,5]$ to social ties $[6,7]$ and others $[8,9]$. Dynamics in multilayer networks have been so far mostly analyzed in situations in which each layer is a fixed network. But even for solitary networks, the node state can evolve while the network itself is changing dynamically, an aspect that still needs to be incorporated into the multilayer framework [10]. In particular we address here coevolution dynamics, that is, coupled dynamics of node states and network topology in which the structure of the network becomes a variable [11-15]. This brings together dynamics of the network with dynamics on the network, going beyond situations of temporal networks decoupled from node state dynamics. Thus coupling layers of coevolution dynamics allows us to model systems where the dynamics and time scales depend on the layer. Examples include social systems where a layer represents a context, e.g., communication channel such as text messages or voice calls [16], or a relationship (family, coworkers, friends) $[6,17,18]$.

Coevolution dynamics in a single-layer network has been considered in a variety of contexts $[14,19]$, including social differentiation [20], neural systems [21], epidemic spreading [22], opinion formation [13,23,24], cultural dynamics [25,26], and ecosystems [27,28]. The rewiring (plasticity) parameter $p$, measuring the relative time scale of evolution of the network and the states of the nodes, is typically the control parameter of coevolution dynamics. There, a generic phenomenon is a fragmentation transition $[13,25]$ that splits the network into disconnected components. This transition occurs at a critical value $p_{c}$ of the rewiring parameter.

The coevolving voter model (CVM) [13] is an archetypal example displaying the fragmentation transition. The state of the system for coevolving networks is characterized through the interface density $\rho$ quantifying the fraction of edges linking nodes with different states (active links). When $\rho \neq 0$, the system is active, while for $\rho=0$ it is frozen, which in finitesize systems happens at finite times. In complex networks and if the rewiring probability $p$ is low enough, a single realization $\rho$ fluctuates around an asymptotic value $\rho^{\text {asym }}$, measured as the $t \rightarrow \infty$ limit of the interface density averaged over active runs at time $t, \rho^{\text {surv }}(t)$ [13]. For $N \rightarrow \infty$ an absorbing transition from an active $\left(\rho^{\text {asym }} \neq 0\right)$ to a frozen $\left(\rho^{\text {asym }}=0\right)$ state occurs at $p=p_{c}$. This transition coincides for finite-size systems with a fragmentation transition of the network freezing into two disconnected components for $p>p_{c}$, each one fully ordered in one of the two possible states. The absorbing transition can be identified using $\rho$, while the fragmentation transition is identified by the relative size of the largest network component $S_{1}$ in the frozen state.

As a prototype situation to describe coevolution dynamics and fragmentation transitions in a multilayer we consider two coupled layers with coevolving voter models. Each layer describes changes of state (for example, opinion) by interactions in a given context with a different topological time scale as characterized by the rewiring parameter of the layer, motivated by [6,16-18]. A key feature of our study is the flexibility of the strength of interlayer connectivity [10,29]. This allows for the existence of nodes present in the two layers as well as other nodes only present in one of the layers. We call $q$, which varies between 0 and 1 , the degree of multiplexing: when $q$ is zero, the system consists of two fully disconnected layers, whereas when $q$ is equal to unity, we have a complete multiplex where all nodes exist in both layers. While $q=1$ corresponds to the current definition of a multiplex [8], the possibility of a partial overlap is a natural framework to study social settings where only a fraction of agents share the same context [29]. We will show that precisely through tuning this multiplexing parameter, we find a shattered fragmentation in coevolution dynamics.

This work is organized as follows. Section II introduces the model. Section III shows that coupling two identical layers into a symmetric multiplex shifts the transition to higher plasticity. Section IV contains the mean-field pair approximation derivation of the evolution of interface densities for a general multiplex, supporting as a special case the result in Sec. III and relating the symmetric multiplex to a monoplex with an effective degree. Section V contains the main results of this work. It shows that in the cases where the rewiring parameters in each layer are different, the more topologically dynamic layer undergoes shattered fragmentation. This phenomenon 


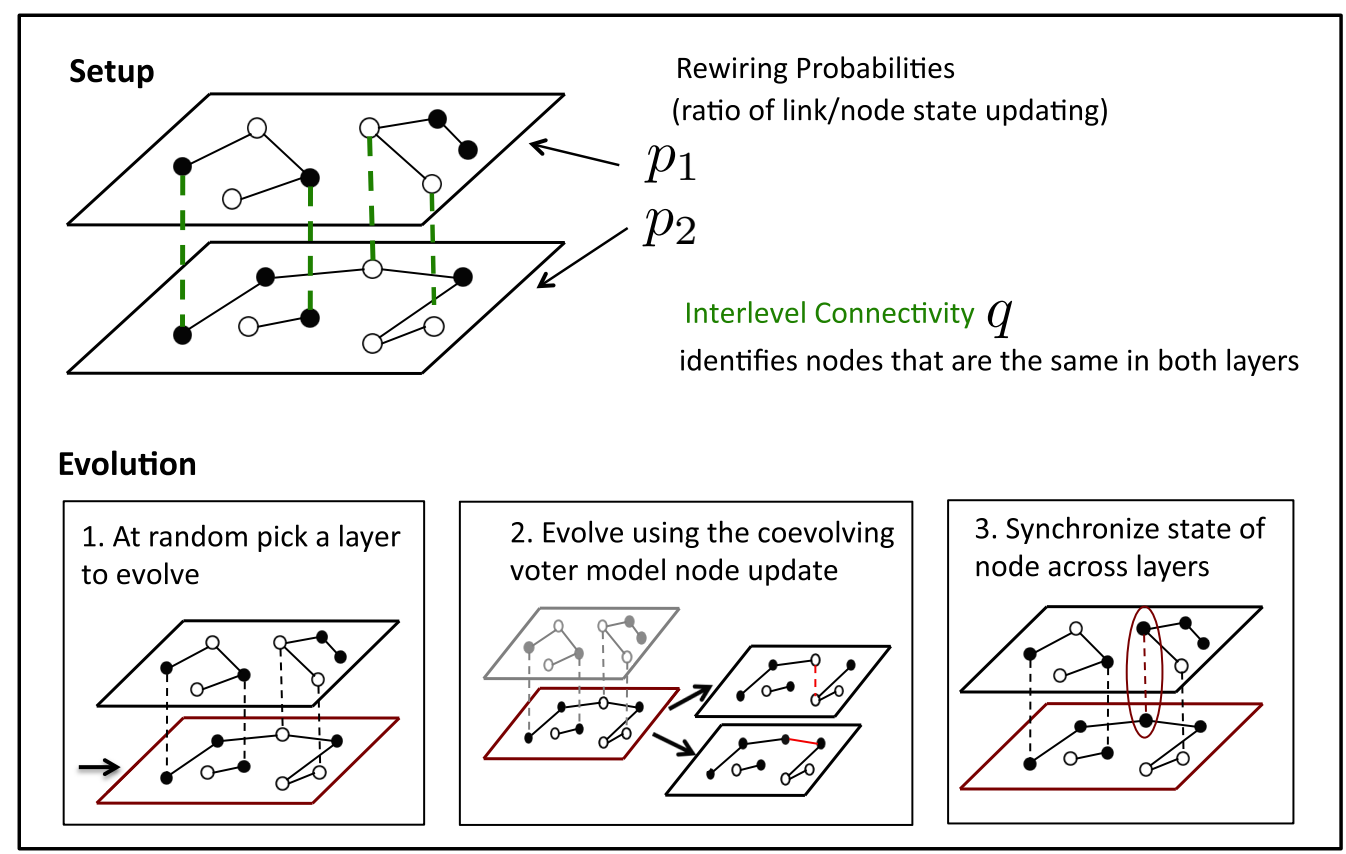

FIG. 1. (Color online) Schematics of two-layer coevolving voter model. The degree of multiplexing is the fraction $q$ of nodes that are identified (connected) across the layers.

is characterized with statistics of components. The work is concluded in Sec. VI.

\section{THE MODEL}

We couple together two binary state CVMs into a multilayer system (Fig. 1). Each layer $l \in\{1,2\}$ contains a network with $N_{l}$ nodes and an average degree $\mu_{l}$, where the state of each node can be \pm 1 . In order to compare with previous results in monolayer networks, we set $N_{1}=N_{2}=N$, and the layers are degree regular networks in the initial condition. The initial states of the network nodes are random and equiprobable. We link the two layers by identifying a proportion $q$ of nodes across layers. Each CVM is characterized by its rewiring $p_{1}$ and $p_{2}$. There are three key parameters in our model (1): the plasticity of each layer (given by $p_{1}$ and $p_{2}$ ) and the degree of multiplexing $q$.

A time step is defined by $N$ updates, where each update involves selecting a random layer and evolving it with CVM rules. Since we require that nodes connected across layers are the same, any change in their states instantly propagates across the layers and changes their interlayer counterpart. To evolve a single CVM network a node $i$ in the selected layer $l$ is randomly selected. Its state is compared to that of a randomly chosen neighbor $j$ (in the same layer), and: nothing happens if the two are the same; otherwise, with probability $1-p_{l}$, node $i$ copies the state of $j$, or else (with probability $p_{l}$ ) it severs the connection with $j$ and draws a link to a node randomly chosen from the set of nodes in layer $l$ that have the same state as $i$ but are not connected to it (if the set is empty, no rewiring is made).

\section{TRANSITION IN A SYMMETRIC MULTILAYER}

The symmetric multilayer system corresponds to $p=p_{1}=$ $p_{2}$. Statistical equivalence of the initial conditions for each layer means that the average density of interfaces shows the same behavior in each layer. For the multilayer voter model ( $p=0$ [30]) the stronger the interlayer connectivity the higher $\rho^{\text {asym }}$ [see Fig. 2(a)]. Thus multiplexing increases the fraction of active links, i.e., the degree of disorder in the system.

The variation of $\rho^{\text {asym }}$ with $p$ is shown in Fig. 2(b). For $q=0$ we recover the absorbing transition of [13]. It continues to exist as two identical layers are interconnected, but now the critical rewiring $p_{c}(q)$ shifts to larger values. This implies that there is a range of rewiring at which disconnected layers would freeze but where any interlayer connection keeps the system active. The degree of multiplexing necessary to achieve this increases monotonically with $p$. This range is finite, i.e., $p_{c}(1)<1$ : if the time scale on which the topology of the system changes is sufficiently large (large $p$ ), even a fully connected multiplex will freeze.

The shift in the absorbing transition is mirrored by the offset in the fragmentation transition [Fig. 2(c)]: the multiplex can sustain a higher rate of rewiring with each layer still freezing into only one connected component. Above $p_{c}(q)$ each layer in the stationary system consists of two components with differing states, with each such component connected to its counterpart in another layer. The characteristic time $\tau$, i.e., the average time at which the multilayer system reaches an absorbing state [Fig. 2(d)], diverges around $p_{c}(q)$ in a critical slowing down that is once more indicative of a fragmentation transition.

\section{PAIR APPROXIMATION FOR INTERFACE DENSITIES}

We use a mean-field pair approximation $[13,31,32]$ scheme to derive equations governing the evolution of interface densities $\left(\rho_{1}, \rho_{2}\right)$ of the two layers in the $N \rightarrow \infty$ limit. Let the multiplex interface densities, or the densities of edges linking nodes with different states, be given by $\rho_{l}$, where $l$ refers to the 

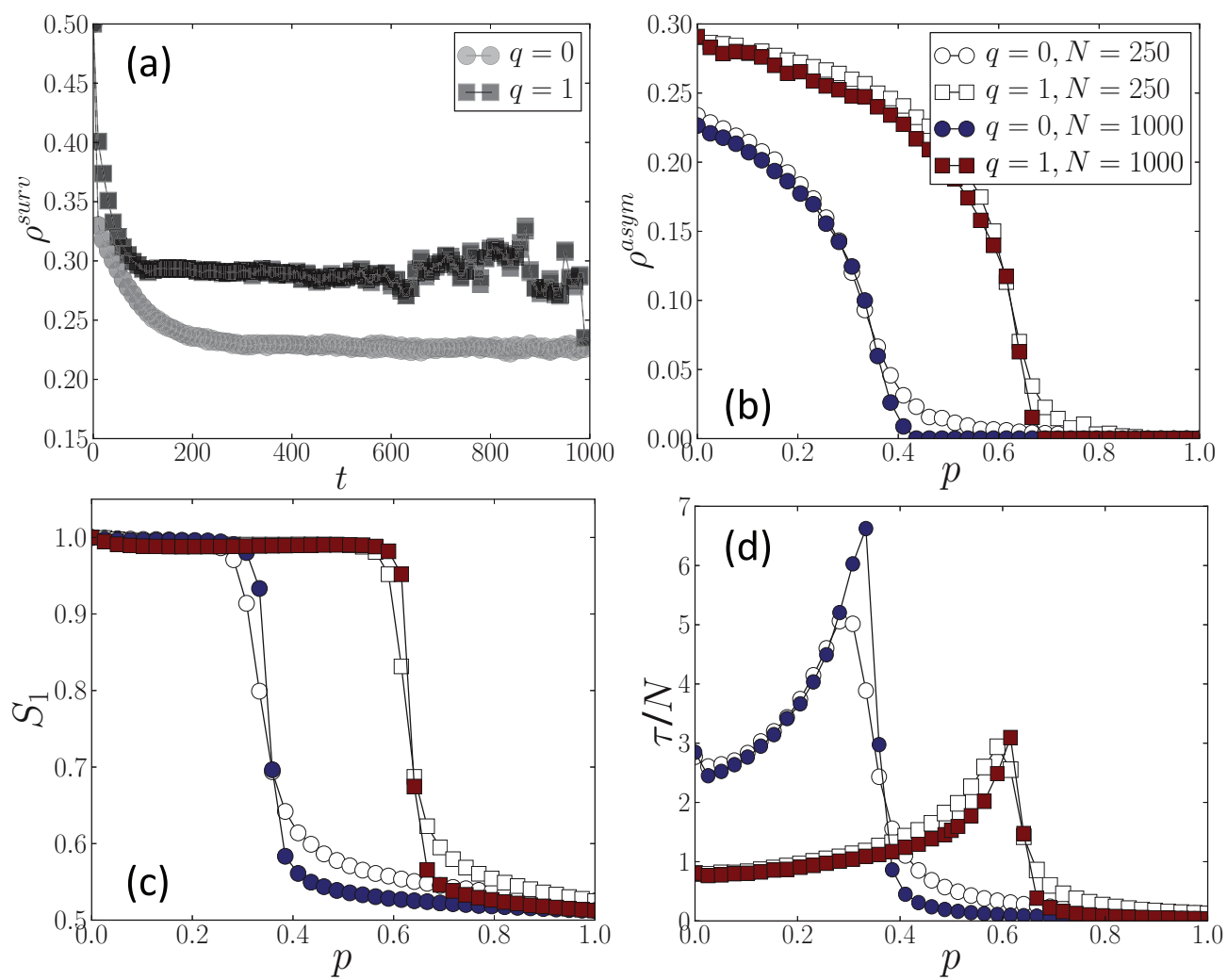

FIG. 2. (Color online) (a) Interface density in a multilayer network of $N=N_{1}=N_{2}=250$ nodes, averaged over realizations still active at time $t$ for $p=p_{1}=p_{2}=0$ and interlayer connectivity $q$. (b) The asymptotic interface density obtained as the plateau in (a). (c) Average size of the largest cluster in the frozen state. (d) Scaled average time to reach an absorbing state. In all setups the ensemble consists of $10^{4}$ realizations. Parameter values for (b)-(d) are shown in (b).

layer. In the two-layer multiplex $l \in\{1,2\}$. We now consider the changes to $\rho_{l}$ for an arbitrary layer.

First, we consider changes to $\rho_{l}$ that come from updating layer $l$ itself. Given that an active link is selected, a node will change state with probability $1-p_{l}$. This would result in the inversion of active and inactive links around that node, giving a change to $\rho_{l}$ of $(k-2 n) / L_{l}$, where $k$ is the number of neighbors of that node in that layer, $n$ is the number of active links it has (in that layer), and $L_{l}$ is the total number of links, which in the random regular networks with mean degree $\mu_{l}$ is given by $L_{l}=\mu_{l} N / 2$ (we take networks in the two layers to have an equal number of nodes, $N_{1}=N_{2}=N$ ). Alternatively, with probability $p_{l}$ that node can rewire, reducing $\rho_{l}$ by a unit of $1 / L_{l}$. Given a node with $n$ out of $k$ links being active, the probability to select an active link is $n / k$. Then, if $B(n, k)$ is the probability of a node with $k$ edges to have $n$ active ones and $P(k)$ is the probability of a node to have $k$ edges [thus $\mu_{l}=\sum_{k} k P_{l}(k)$ ], then summing all these gives the changes to $\rho_{l}$ stemming from an update of layer $l$ as

$$
\dot{\rho}_{l}^{\text {layer } l}=\sum_{k} P_{l}(k) \sum_{n=0}^{k} B_{l}(n, k) \frac{n}{k}\left(\left(1-p_{l}\right) \frac{k-2 n}{L_{l}}-p_{l} \frac{1}{L_{l}}\right) .
$$

In addition to that, the change in $\rho_{l}$ can come about as a result of a change of state at another layer $m \neq l$ that propagates to layer $l$. This propagation will happen with probability $q$, which is the fraction of nodes connected across the layers. The contribution from an update of layer $m$ is given by

$$
\begin{aligned}
\dot{\rho}_{l}^{\operatorname{layer} m}= & q \sum_{k} P_{m}(k) \sum_{n=0}^{k} B_{m}(n, k) \frac{n}{k}\left(1-p_{m}\right) \\
& \times\left[\sum_{k} P_{l}(k) \sum_{n=0}^{k} B_{l}(n, k) \frac{k-2 n}{L_{l}}\right] .
\end{aligned}
$$

Here another double sum is needed to include all the possible changes in layer $l$.

We now assume that for both layers the mean-field scenario holds, so that $B_{l}$ is distributed binomially where for a given $k, \sum_{n=0}^{k} n B_{l}(n, k)=k \rho_{l}$, and $\sum_{n=0}^{k} n^{2} B_{l}(n, k)=$ $k \rho_{l}+\rho_{l}^{2} k(k-1)$ (the same for $B_{m}$ ). We take the networks to be uncorrelated. Equations (1) and (2) can be combined, where each contribution comes with an additional factor of $1 / 2$ that reflects the equiprobable selection of the initial update layer, as well as a time rescaling of $1 / N$ to make it comparative to the monoplex case, i.e.,

$$
\dot{\rho}_{l}=\frac{N}{2}\left[\dot{\rho}_{l}^{\text {layer } l}+\dot{\rho}_{l}^{\operatorname{layer} m}\right]
$$

The result is easily written in terms of the variables $A_{l}=$ $\frac{1}{\mu_{l}}\left(1-p_{l}\right)\left(\mu_{l}-1\right)$ and $B_{l}=q\left(1-p_{l}\right)$ :

$$
\dot{\rho}_{l}=-2 A_{l} \rho_{l}^{2}+\left(A_{l}-\frac{1}{\mu_{l}}\right) \rho_{l}+B_{m} \rho_{m}\left(1-2 \rho_{l}\right) .
$$


Thus $B_{l}$ quantifies the effect of the other layer on the interface density (note that there is no interlayer influence for either $q=0$ or only rewiring $p_{l}=1$ ).

Let us now examine the special case of the symmetric multiplex, by which we mean a two-layer system with equal rewiring probabilities, i.e., where $p_{1}=p_{2}=p$. Throughout the paper we also select the networks for each layer from the same ensemble defined by some $\mu=\mu_{1}=\mu_{2}$. Hence the above variables become $B_{1}=B_{2}=q(1-p)$ and $A_{1}=A_{2}=$ $1 / \mu(1-p)(\mu-1)$.

Making a further simplification, we assume the same initial condition $\rho_{1}(0)=\rho_{2}(0)$ on each of the layers. In all the cases studied the binary states will be equidistributed, making the initial interface densities 0.5 for each layer. Since the evolution equations are the same for each layer, $\rho_{1}(t)=\rho_{2}(t)=\rho(t)$, and Eq. (3) can be written as

$$
\dot{\rho}=\frac{\rho}{\mu}\{(1-p)(1-2 \rho)[\mu(q+1)-1]-1\} .
$$

Hence if $\mu_{\mathrm{eff}}=\mu(q+1)$, then Eq. (4) becomes

$$
\dot{\rho}=\frac{\rho}{\mu}\left[(1-p)\left(\mu_{\mathrm{eff}}-1\right)(1-2 \rho)-1\right] .
$$

In [13] the evolution of $\rho$ for the monolayer is given by

$$
\dot{\rho}_{\text {mono }}(\mu)=\frac{2 \rho}{\mu}[(1-p)(\mu-1)(1-2 \rho)-1] .
$$

Hence the multilayer equation (5) can be written as

$$
\dot{\rho}(\mu)=\frac{1+q}{2} \frac{2 \rho}{\mu_{\mathrm{eff}}}\left[(1-p)\left(\mu_{\mathrm{eff}}-1\right)(1-2 \rho)-1\right],
$$

or

$$
\dot{\rho}(\mu)=\frac{1+q}{2} \dot{\rho}_{\mathrm{mono}}\left(\mu_{\mathrm{eff}}\right) .
$$

Hence the evolution of the interface density of the symmetric multiplex is the same as that of a monoplex with a certain effective degree but with an effective rescaling of time by $\frac{1+q}{2}$. In other words, the evolution of $\rho$ for the multiplex is equivalent to that of the monoplex but with

$$
\begin{aligned}
& \mu \rightarrow \mu_{\mathrm{eff}}=\mu(1+q), \\
& d t \rightarrow d t_{\mathrm{eff}}=\frac{2}{1+q} d t .
\end{aligned}
$$

When $q=0$, the multiplex evolves twice as slow as the single network, but that is because in one time step, on average, only half of each layer would be updated. (We have $N$ updates per time step, and two layers of $N$ nodes each. The monoplex has one layer with $N$ nodes and evolves with $N$ updates in a time step.) When $q=1$, the multiplex evolves as fast as, and is identical to, a single network with twice the mean degree. We also note that the asymptotics (fixed points of $\rho$ ) of the multiplex are given by the monoplex with an appropriately rescaled degree.

Specifically, the stationary interface density for the multilayer voter model $(p=0)$ is given by

$$
\rho^{*}=\frac{\mu_{\mathrm{eff}}-2}{2\left(\mu_{\mathrm{eff}}-1\right)}=\frac{\mu(1+q)-2}{2[\mu(1+q)-1]},
$$

in agreement with the numerical simulations [Fig. 2(a)] [33]. For arbitrary $p$ the stationary interface density surface $\rho^{*}(p, q)=\rho_{1}^{*}=\rho_{2}^{*}$ is illustrated in Fig. 3(a). The system displays a phase transition at a critical rewiring probability $p_{c}(q)$ that increases with the degree of multiplexing $q$. This corresponds to the increase in the effective degree and is supported by the numerical results [Fig. 2(b)].

For arbitrary $\left(p_{1}, p_{2}\right)$ there are four fixed point solutions $\left(\rho_{1}^{*}, \rho_{2}^{*}\right)$, including $(0,0)$, with at most one such that $\left(\rho_{1}^{*}>\right.$ $0, \rho_{2}^{*}>0$ ). If such a solution exists, it is stable and corresponds to a fully active system with finite interface densities. In this case the $(0,0)$ origin is necessarily unstable, and hence the existence of an active state can be checked by examining the stability of $(0,0)$. Three parameter cross sections at three different $q$ values are shown in Fig. 3(b). The multilayer dynamics exhibits three phases: both layers active, both frozen, and a mixed phase when one layer is active but the other is not. This latter phase exists only for a completely disconnected system, so that any degree of multiplexing $(q \neq 0)$ is enough

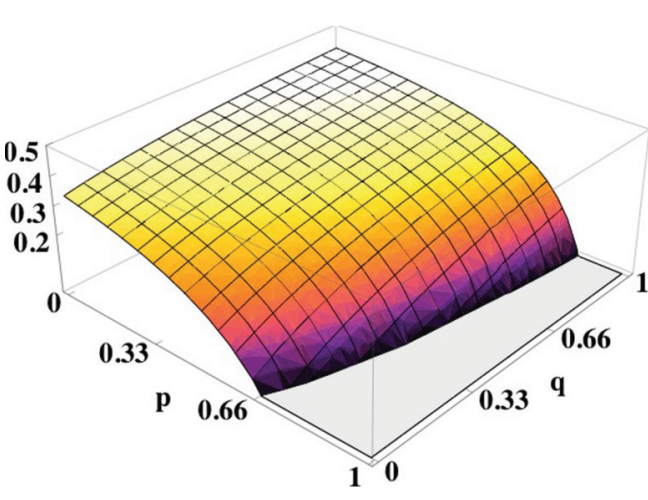

(a) Stable internal solution
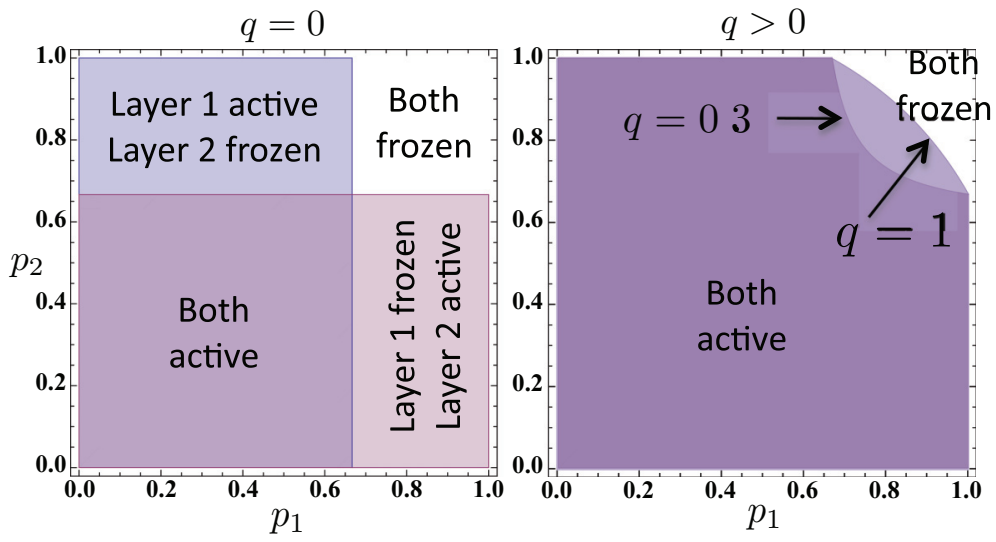

(b) Layer activity in $\left(p_{1}, p_{2}, q\right)$

FIG. 3. (Color online) (a) Interface density $\rho^{*}$ for the symmetric multiplex with $p=p_{1}=p_{2}$ [given by Eq. (3)]. (b) Stability analysis of the $\left(\rho_{1}, \rho_{2}\right)=(0,0)$ fixed point. For $q>0$ both layers are active when $(0,0)$ is either unstable or saddle; the system is frozen when $(0,0)$ is stable. 

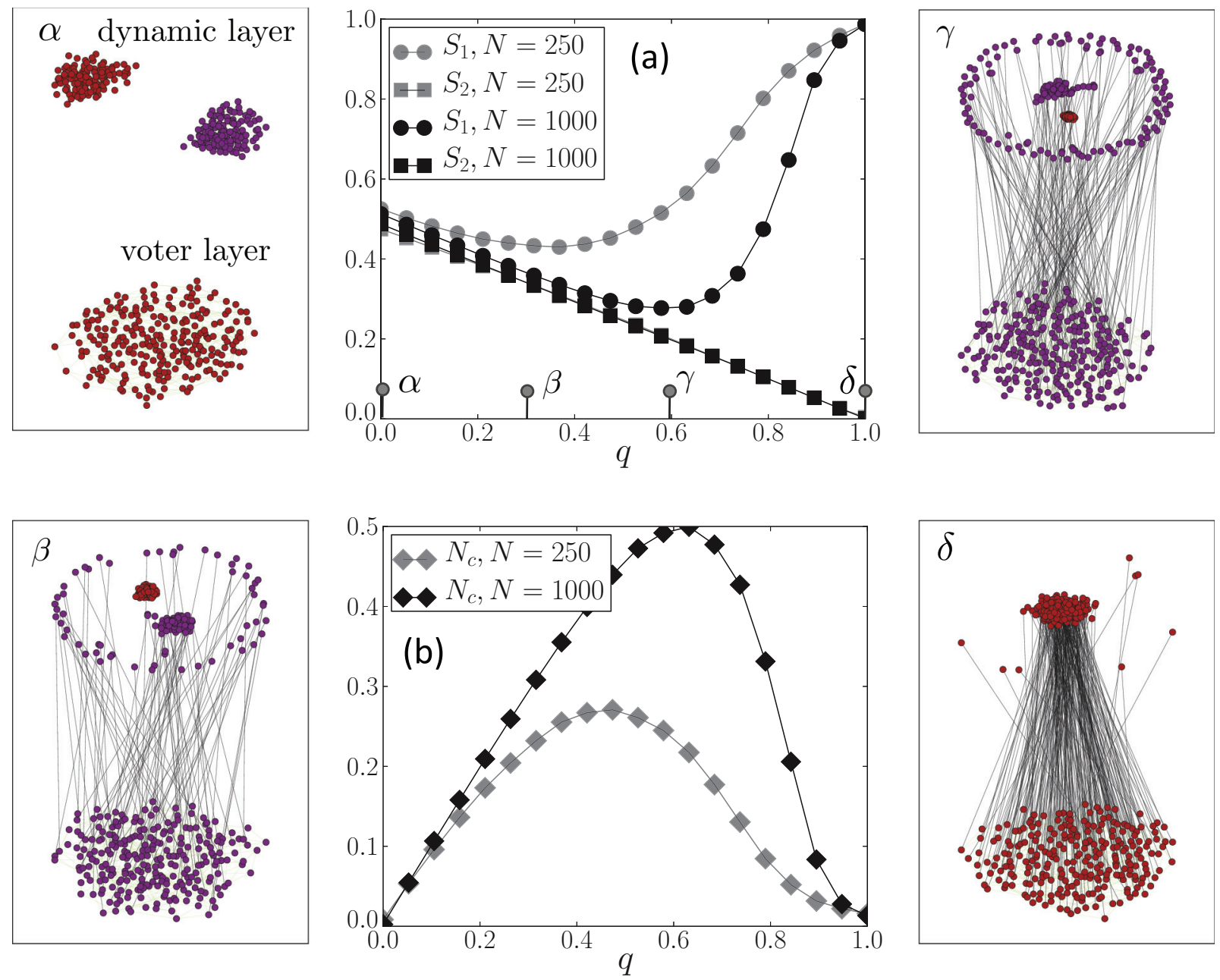

FIG. 4. (Color online) (a) Relative size of the $i$ th largest components $S_{i}$ in the dynamic layer $\left(p_{1}=1\right)$ coupled with strength $q$ to the voter layer $\left(p_{2}=0\right)$ for systems with $N$ nodes in each layer. (b) Relative number of connected components $N_{c}$ in the dynamic layer. Variables are averaged over $10^{4}$ frozen configurations. The sides panels show typical snapshots of the $N=250$ system for four sample values of $q$.

to tie the fate of one layer to that of another. In Fig. 3(b) the stable regime thus corresponds to the both-layers-frozen phase, while the both-layers-active phase happens for both the unstable and saddle $(0,0)$, as long as the layers are connected. In the asymmetric case $\left(p_{1} \neq p_{2}\right)$ the stable internal fixed point need not be located on the diagonal, meaning that the activity of the two layers need not be equal.

\section{ANOMALOUS SHATTERED FRAGMENTATION IN THE ASYMMETRIC MULTIPLEX}

The extreme asymmetry scenario couples a layer that only changes states ( $p=0$, which we call the voter layer) and a layer that only rewires ( $p=1$, the dynamic layer). Hence the voter layer is not affected by the dynamic layer. Instead, it acts as a driver of the other layer and thus does not fragment for any $q$ [34]. For intermediary multiplexing, the dynamic layer displays an explosion in the number of disconnected nodes as a precursor of an anomalous fragmentation transition that we call shattered fragmentation (Fig. 4). Only two network components are ever significant in that layer, the rest being isolated nodes. For increasing $q$ the dynamic layer shows (i) an increasing number of isolated components [which correspond to almost $q N$ nodes connected to the voter layer, Fig. 4(b)], (ii) the second largest component composed of nodes disconnected from the voter layer that were initially in the state opposite to that reached by the voter layer, i.e., $S_{2}=$ $1 / 2(1-q)$, and (iii) the largest component formed by the remaining nodes. For larger $q$ its size $S_{1}$ increases until there is only one connected component left in that layer, which happens at $q=1$.

In the limit of infinite system size $S_{1}$ tails $S_{2}$ for a longer region of $q$, until $q^{*}$, which is defined as the minimum interlayer connectivity that realizes $S_{1}\left(q^{*}\right)=1$, where $S_{1}(q)=$ $\lim _{N \rightarrow \infty} S_{1}(q, N)$. We identify $q^{*}$ with a critical degree of multiplexing, or the minimum interlayer connectivity necessary to stop the dynamic layer from fragmenting. For extreme asymmetry, $q^{*}\left(p_{1}=1, p_{2}=0\right)=1$, meaning that as long as $q<1$, it is impossible to prevent fragmentation of an infinitely large system for these parameters.

Shattered fragmentation is a general consequence of the rewiring asymmetry $p_{1} \neq p_{2}$. Figure 5 quantifies it in terms of $\Delta S=S_{1}-S_{2}$ and $N_{c}: \Delta S$ informs on the existence of a fragmentation transition, and $N_{c}$ informs on the nature of fragmentation. Figure 5 illustrates two ways of varying asymmetry: lowering or raising the rewiring of one layer 


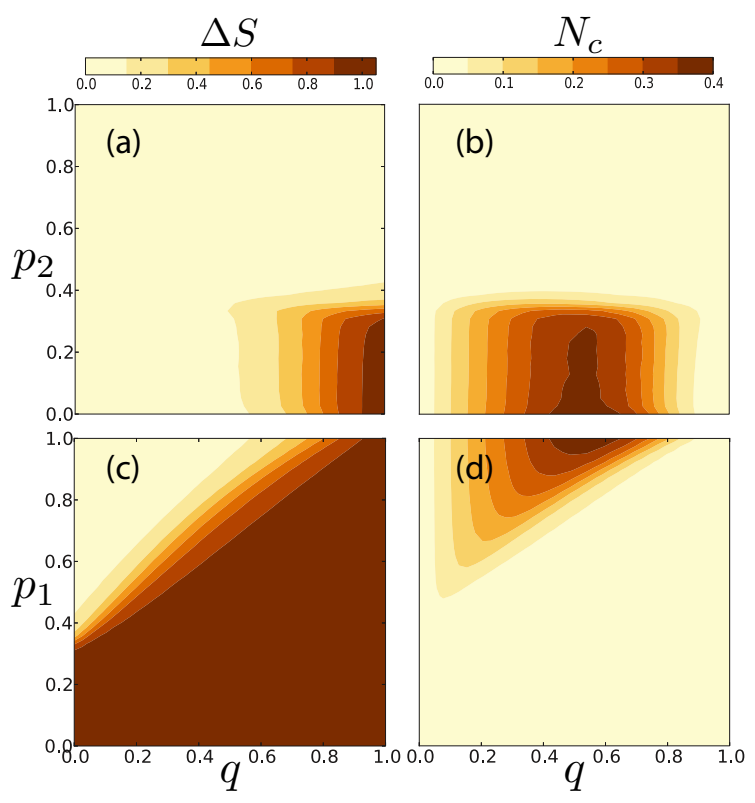

FIG. 5. (Color online) Shattered fragmentation of layer 1 in terms of (left) the difference in the relative size of its two largest components $\Delta S=S_{1}-S_{2}$ and (right) the relative number of connected components $N_{c}$, with (a) and (b) $p_{1}=1$ and (c) and (d) $p_{2}=0$. An unfragmented layer is dark in $\Delta S$. Standard two-component fragmentation (light $N_{c}$ ) becomes shattered as the number of isolated nodes increases (any darkening in $N_{c}$ ). Quantities are averaged over $10^{4}$ realizations of the system with $N=500$ nodes in each layer. A higher system size would have sharper transitions in $\Delta S$ and higher peaks in $N_{c}$ that also move to higher values of $q$.

while keeping the rewiring of the other layer fixed. As long as the states of the nodes of the more dynamic layer are allowed to change $(p \neq 1)$, its fragmentation can be prevented by coupling it to a layer that, uncoupled, would not fragment. This corresponds to a master-slave coupling. For any $\left(p_{1}, p_{2}\right), \Delta S(q)$ displays a steeper transition for increasing $N$, suggesting a step transition in the thermodynamic limit (Fig. 6 ). The fact that fragmentation in one layer need not necessarily entail fragmentation in another is not a feature of extreme asymmetry (Fig. 7). The critical degree of multiplexing decreases from extreme asymmetry $q^{*}\left(p_{1}=1, p_{2}=0\right)=1$ to symmetry $q^{*}\left(p_{1}=0.5, p_{2}=0.5\right)=0.5$ (Fig. 6), decreasing for smaller rewiring. When the (more) static layer is the voter model, $q^{*}\left(p_{1}>p_{c}(0)\right)$ follows a quasilinear dependence on $p_{1}$. On the other hand, for $N \rightarrow \infty, q^{*}\left(p_{1}=1, p_{2}\right) \rightarrow 1$. Fragmentation of the (more) dynamic layer is maximized by asymmetry, where it shatters the layer into isolated components. Their fraction decreases as the layer becomes more static [Fig. 5(d)] but is not dependent on the exact extent of the rewiring of the stabilizing layer [Fig. 5(b)].

Finally, we note that the absorbing and fragmentation transitions coincide in simulations of even the asymmetric multiplex, just as in solitary CVM. Therefore we associate $q^{*}$ with the minimal multiplexing necessary to keep the more dynamic layer active. The pair approximation fails to capture the anomalous transition since the nature of shattered fragmentation is indicative of the presence of isolated nodes. Thus analytics suggest that even minimal multiplexing is sufficient to keep the dynamic level active and does not take into account that the flux of links away from the interconnected nodes might still keep it topologically frozen.

\section{SUMMARY AND CONCLUSIONS}

We have analyzed a multilayer system constructed by coupling together with an arbitrary degree of multiplexing $q$ two coevolving networks with different rewiring parameters. The multilayer structure offsets the critical value of the rewiring for the occurrence of absorbing and fragmentation transitions; hence multiplexing is shown to be able to prevent network fragmentation. We have also found a critical degree of multiplexing characterized as the minimal required interlayer connectivity necessary to stop the fragmentation of a layer by coupling it to a layer that does not fragment. This critical
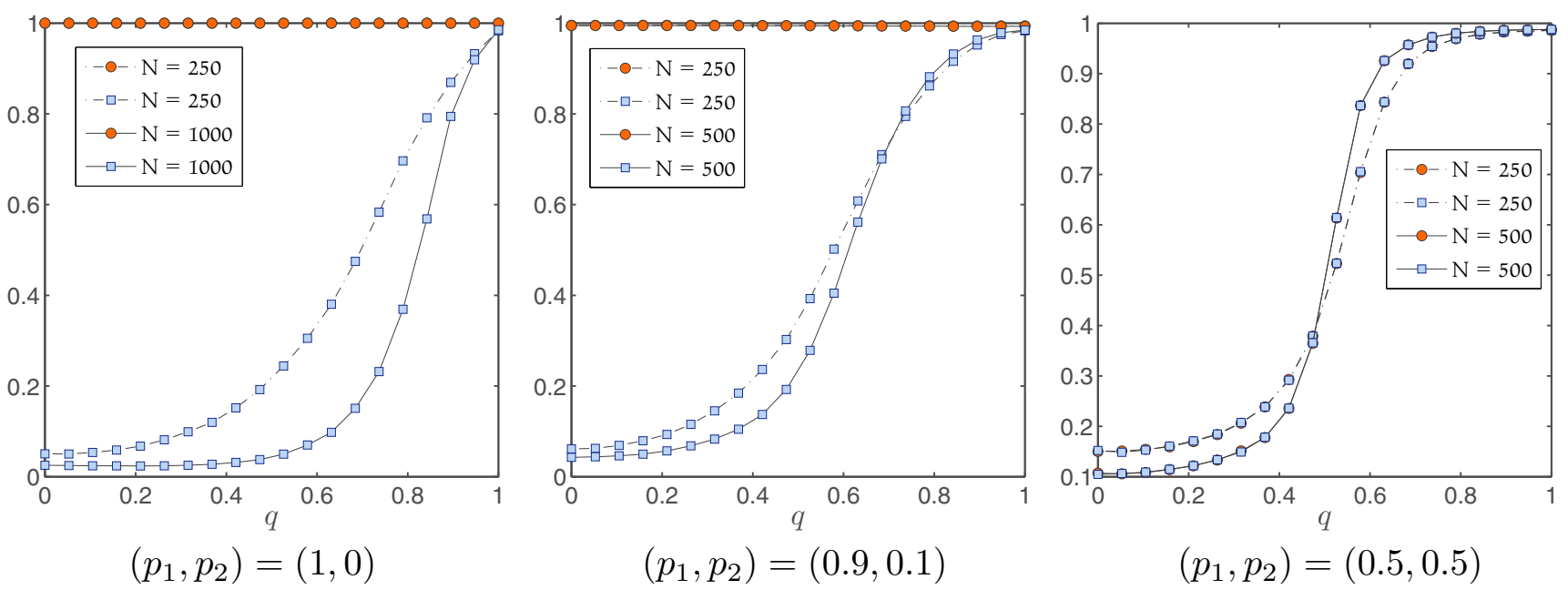

FIG. 6. (Color online) Difference $\Delta S$ between the two largest clusters in each layer (layer 1 in blue, layer 2 in orange) for different sample sizes $N$ and rewiring probabilities $\left(p_{1}, p_{2}\right)$ as a function of interlayer connectivity $q$. Cluster sizes are averaged over $10^{4}$ configurations of systems upon reaching the frozen state. 


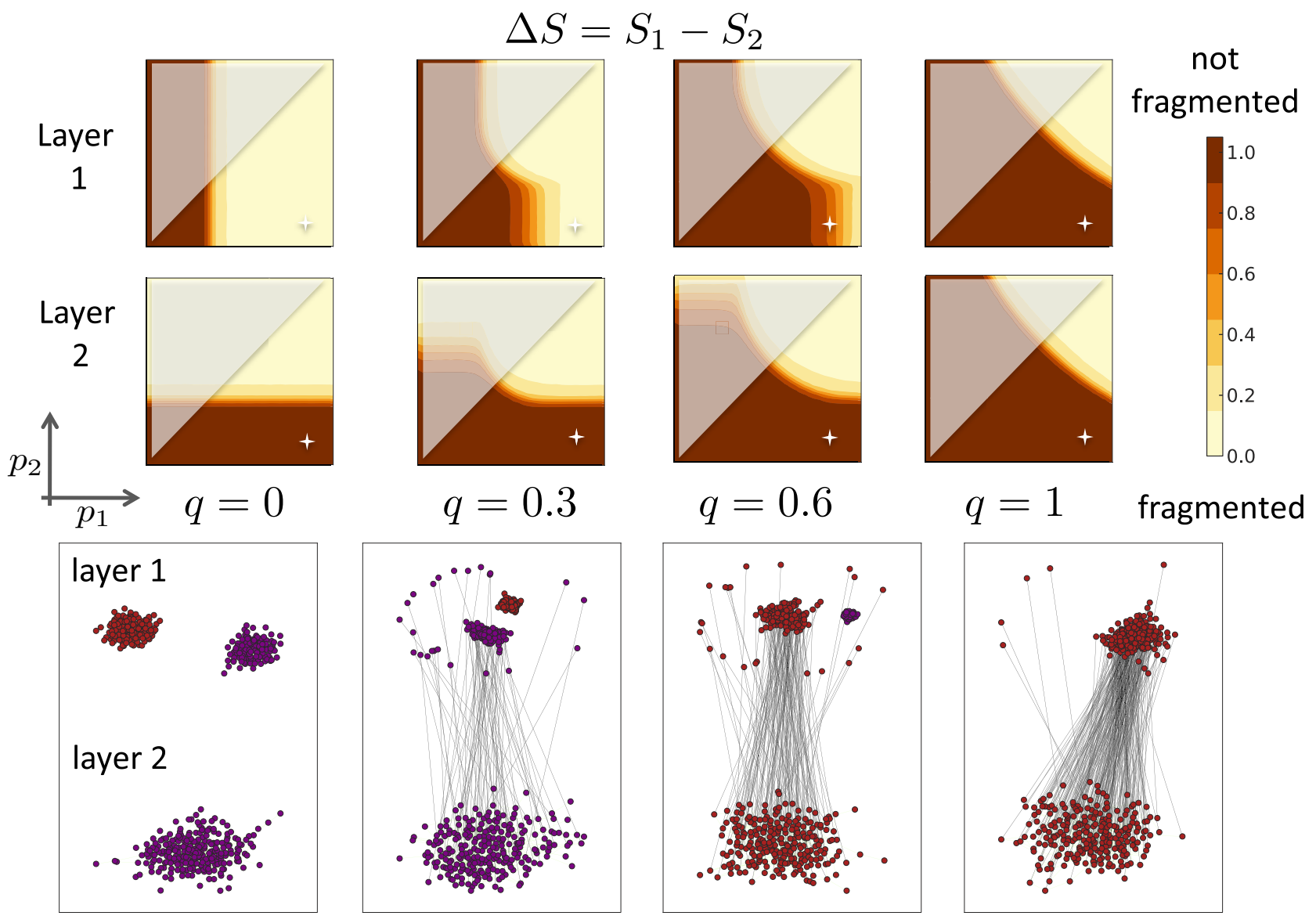

FIG. 7. (Color online) (top) Difference $\Delta S$ between the two largest clusters in layer 1 (first row) and in layer 2 (second row). Due to the symmetry in rewiring rates, the second row is a symmetric transformation of the first, although for ease of reading both are shown together, and only the bright bottom triangles should be looked at. The squares correspond to $\left(p_{1}, p_{2}\right)$ cross sections of the parameter hypercube at various $q$ values. Each point is an average over $10^{4}$ realizations of a multiplex with $N=500$ nodes on each layer. The color scheme ranges between the prevalent colors observed, with light corresponding to 0 and dark to 1. (bottom) Snapshot of a typical absorbing state computed at the starred point in the rows above, $\left(p_{1}, p_{2}\right)=(0.9,0.1)$.

value is a function of the rewiring parameters of the two layers. Subcritical multiplexing leads to the existence of a shattered fragmentation typical of the more topologically dynamic layer as a consequence of dynamic asymmetry between the layers. Our findings suggest that the opinion formed in situations that display significant variation in terms of flexibility of connections can leave a large number of agents disconnected from each other in settings where links are more easily broken. Such theoretical indications of a shattered fragmentation, not defined solely by the size of the largest component, are particularly pertinent to qualify the nature of fragmentation possibly observed in multilayer systems. Moreover, the shat- tered fragmentation resulting in an explosion of isolated nodes as the degree of multiplexing approaches its critical value is not captured by a pair approximation calculation. Other approaches should be explored to handle analytically the growth of isolated nodes [32].

\section{ACKNOWLEDGMENTS}

This work has been supported by the Spanish MINECO and FEDER under projects INTENSE@COSYP (FIS2012-30634) and MODASS (FIS2011-24785) and by the EU Commission through the project LASAGNE (FP7-ICT-318132).
[1] M. De Domenico, A. Solé-Ribalta, E. Cozzo, M. Kivelä, Y. Moreno, M. A. Porter, S. Gómez, and A. Arenas, Phys. Rev. X 3, 041022 (2013).

[2] M. Kivelä, A. Arenas, M. Barthelemy, J. P. Gleeson, Y. Moreno, and M. A. Porter, arXiv:1309.7233.

[3] S. V. Buldyrev, R. Parshani, G. Paul, H. E. Stanley, and S. Havlin, Nature (London) 464, 1025 (2010).
[4] C. Granell, S. Gómez, and A. Arenas, Phys. Rev. Lett. 111, 128701 (2013).

[5] F. Radicchi and A. Arenas, Nat. Phys. 9, 717 (2013).

[6] M. Szell, R. Lambiotte, and S. Thurner, Proc. Natl. Acad. Sci. USA 107, 13636 (2010).

[7] A. Halu, K. Zhao, A. Baronchelli, and G. Bianconi, Europhys. Lett. 102, 16002 (2013). 
[8] S. Gómez, A. Díaz-Guilera, J. Gómez-Gardeñes, C. J. PérezVicente, Y. Moreno, and A. Arenas, Phys. Rev. Lett. 110, 028701 (2013).

[9] Z. Wang, A. Szolnoki, and M. Perc, Sci. Rep. 3, 2470 (2013).

[10] S. Shai and S. Dobson, Phys. Rev. E 87, 042812 (2013).

[11] M. G. Zimmermann, V. M. Eguíluz, and M. San Miguel, in Economics with Heterogeneous Interacting Agents (Springer, Berlin, 2001), pp. 73-86.

[12] M. G. Zimmermann, V. M. Eguíluz, and M. San Miguel, Phys. Rev. E 69, 065102 (2004).

[13] F. Vazquez, V. M. Eguíluz, and M. San Miguel, Phys. Rev. Lett. 100, 108702 (2008).

[14] T. Gross and B. Blasius, Proc. R. Soc. Interface 5, 259 (2008).

[15] C. Castellano, S. Fortunato, and V. Loreto, Rev. Mod. Phys. 81, 591 (2009).

[16] A. Stopczynski, V. Sekara, P. Sapiezynski, A. Cuttone, M. M. Madsen, J. E. Larsen, and S. Lehmann, PLoS ONE 9, e95978 (2014).

[17] P. Klimek and S. Thurner, New J. Phys. 15, 063008 (2013).

[18] P. Csermely, Talent Dev. Excellence 5, 115 (2013).

[19] H. Sayama, I. Pestov, J. Schmidt, B. J. Bush, C. Wong, J. Yamanoi, and T. Gross, Comput. Math. Appl. 65, 1645 (2013).

[20] V. M. Eguíluz, M. G. Zimmermann, C. J. Cela-Conde, and M. San Miguel, Am. J. Sociol. 110, 977 (2005).

[21] C. Meisel, A. Storch, S. Hallmeyer-Elgner, E. Bullmore, and T. Gross, PLoS Comput. Biol. 8, e1002312 (2012).
[22] T. Gross, C. J. Dommar D'Lima, and B. Blasius, Phys. Rev. Lett. 96, 208701 (2006).

[23] I. D. Couzin, C. C. Ioannou, G. Demirel, T. Gross, C. J. Torney, A. Hartnett, L. Conradt, S. A. Levin, and N. E. Leonard, Science 334, 1578 (2011).

[24] G. Zschaler, G. A. Böhme, M. Seißinger, C. Huepe, and T. Gross, Phys. Rev. E 85, 046107 (2012).

[25] F. Vazquez, J. C. González-Avella, V. M. Eguíluz, and M. San Miguel, Phys. Rev. E 76, 046120 (2007).

[26] D. Centola, J. C. González-Avella, V. M. Eguíluz, and M. San Miguel, J. Conflict Resolut. 51, 905 (2007).

[27] U. Dieckmann and M. Doebeli, Nature (London) 400, 354 (1999).

[28] M. Cantor and H. Whitehead, Philos. Trans. R. Soc. B 368, 1618 (2013).

[29] C. Buono, L. G. Alvarez-Zuzek, P. A. Macri, and L. A. Braunstein, PLoS ONE 9, e92200 (2014).

[30] R. A. Holley and T. M. Liggett, Ann. Probab. 3, 643 (1975).

[31] F. Vazquez and V. M. Eguíluz, New J. Phys 10, 063011 (2008).

[32] G. Demirel, F. Vazquez, G. A. Böhme, and T. Gross, Phys. D (Amsterdam, Neth.) 267, 68 (2014).

[33] Note that according to Ref. [31], $\rho^{\text {asym }}=2 / 3 \rho$.

[34] As long as one of the layers only rewires $p=1$, the other layer behaves as if it were disconnected and thus fragments at $p_{c}(q=0)$. 